\section{Multidrug resistance of uropathogens at governmental hospitals in the Gaza strip/Palestine}

Sameh A. Alkhodari ${ }^{1}$, Abdelraouf

A. Elmanama ${ }^{2}$

1 HBK Hospital for Rehabilitation and Artificial Limb-Gaza Gaza, Palestine.

2 Medical Laboratory Sciences

Department, Islamic University of Gaza, Gaza strip, Palestine.

\section{Contact information:} wide. Escherichia coli, E. coli and Klebsiella spp. are among the main etiologic for UTI in the Gaza Strip. The growing variations in resistance patterns among uropathogens to antimicrobials is multifactorial and varies globally. It greatly reduces/limits or complicates treatment options.

Aims: To determine the pattern of antimicrobial resistance and multidrug resistance among uropathogens at governmental hospitals.

Methods: We analyzed the data of 11,890 urine samples processed in governmental hospitals in the Gaza Strip, Palestine during 2019. The percentage of resistance wascalculated for uropathogens, and then multidrug resistance was calculated according to Centers for Disease Control and Prevention "CDC" definition.

Results: Of 11,890 urine samples, 2910 (24.5\%) showed significant growth. Escherichia coliwas most frequently isolated (1743; 59.9\%), followed by Klebsiella spp. (725; 24.9\%), Pseudomonas spp. (123; 4.2\%), Streptococcus spp. (98; 3.4\%), Staphylococcus aureus (41; $1.4 \%)$. Microorganisms' resistance was high against ampicillin (92.4\%) and amoxicillin (91.1\%), trimethoprim/sulfamethoxazole (68.2\%), cefalexin $(64.9 \%)$, doxycycline $(61.9 \%)$, nalidixic acid (53.6\%), cefuroxime $(53.0 \%)$, ceftriaxone $(48.9 \%)$, ceftazidime $(43.1 \%)$, ciprofloxacin $(36.9 \%)$, gentamicin $(25.8 \%)$, amikacin $(3.2 \%)$. The resistance of microorganisms in males is higher than females. Multidrug resistance was detected in $37 \%$ of E. coli and $53 \%$ in Klebsiella spp.

Conclusion: Resistance is high and variable among uropathogens isolated from patients in the Gaza strip. Both age and gender are risk factors in both infection and resistance pattern. The multidrug resistance percentage is growing remarkably in the Gaza Strip.

\section{Prof. Dr. Abdelraouf A. Elmanama.}

Address: Medical Laboratory Sciences Department, Islamic University of Gaza, Gaza strip, Palestine.

Đ elmanama_144@yahoo.com

\section{Keywords}

Uropathogens; Resistance;

Urinary Tract Infection;

Multidrug Resistance;

Gaza Strip; Palestine. 


\section{Introduction}

Urinary tract infection (UTI) is a public health problem, in both community and hospitals. It is more prevalent in women than men [1]. According to the American college of obstetricians and gynecologists, about $50-60 \%$ of the women suffer from an episode of UTI at least once during their lifetime [2]. Unmanaged UTI may results in a wide range of serious complications starting from kidney damage, renal scarring and my ends with renal failure. In men, UTI become increasingly frequent with age and functional disability, but bacteriuria in elderly men tends to be intermittent, episodic, and more complex to be diagnosed [3].

Gram-negative bacteria are the most common causative agents of UTI, including E. coli, Klebsiella spp., and Proteus spp. While Gram-positive bacteria constitutes the minority of UTI infectious pathogens like Staphylococcus saprophyticus, Enterococci spp., and Streptococcus spp. [4, 5]. Antimicrobial resistance is becoming a major concern not only in nosocomial complicated UTI, but also in uncomplicated community-acquired UTI [6]. Concurrent resistance to antimicrobials of different structural classes has arisen in several of bacterial species and may complicate the therapeutic management of infections, including those of the urinary tract [7]. Multiple drug resistance (MDR) rate for 3 and more drugs is arising in E. coli isolates and often complicate therapeutic management of urinary tract infection (UTI) by this organism [7]. The extent of the problem in low-and middle-income countries is unknown because of the scarcity of data, but high levels of resistance are increasingly being reported worldwide [8].

In Palestine, antimicrobials are poorly regulated and available on the private market and can be accessed with or without a prescription [9]. Gaza Strip is one of the high-density populated area, in which governmental hospitals is the back bone of UTI infections treatment. In this study, the common uroptahogens were determined and the pattern of resistance of E. coli and Klebsiella spp. were detected and the MDR rates were determined.

\section{Methods and materials}

\section{Study design and settings}

This is a retrospective study in design that was conducted in four governmental hospitals (European, Al Shifa, Nassr, and Al Durra Hospitals) in Gaza Strip, Palestine over a period one year (1/1/2019 to 31/12/2019).

\section{Data collection}

The study data were collected from urine culture records of samples that were sent to the Microbiology laboratory during the study period. All samples diagnosed with UTI infection by physicians in hospital were included. An approval from the Helsinki Ethical Committee was obtained before the study and a permission from the Ministry of Health was granted to use the data.

The records of 11,890 urine samples results were studied. According to the microbiology lab Standard Operation Procedure, midstream urine samples were collected in sterile containers and cultured on blood agar and MacConkey media with a standard calibrated loop and were incubated at $37^{\circ} \mathrm{C}$ overnight. A growth of $\geq 10^{5}$ colony-forming units/ $\mathrm{mL}$ was considered as significant bacteriuria. The identification of isolates was done through Gramstaining and conventional biochemical methods. Antimicrobial susceptibility was done by Kirby-Bauer disc diffusion method on Mueller-Hinton agar and the interpretations were carried out according to the Clinical and Laboratory Standards Institute guidelines [10]. Antibiotic disks used in the susceptibility testing varied according to their availability. Data were obtained in the form of Microsoft excel file containing all the recorded cases UTI cultures results contains susceptibility, resistance to the used antimicrobials, name of the uropathogen, age, gender, date of test, area of living, name of hospital, and diagnosis at the 
Vol. 11 No. 1:4 doi: $10.3823 / 855$ four governmental hospitals, the data cleaned, coded and exported to SPSS to be analyzed.

\section{Data analysis}

Data analysis was done, using SPSS 22 (2019). Cross tabulation was made; to detect any correlations, and/or differences among the investigated variables, using chi square, and independent $T$ test. P-value equal or less than 0.05 was considered statistically significant, with confidence interval (CI) of 95\%. Tables, histograms, and charts were used to present the data. Indicators which used in the study designed to reflect antimicrobial susceptibility situation in governmental hospitals.

\section{Results}

\section{Distribution frequency of uropathogens}

Only 2,910 (24.5\%) exhibited significant growth and were considered as positive for UTI. Among the uropathogens isolated during the data collection period, E. coli constituted the majority with $59.9 \%$, followed by Klebsiella spp. (24.9\%), Pseudomonas spp. (4.2\%), and Proteus spp. (2.4\%). Gram-negative bacteria constituted $91 \%$ of the isolates, while Gram-positive (S. aureus, Enterococcus spp., and Streptococcus spp.) represented in less than $5.5 \%$.In addition, less than $3 \%$ of the isolates were classified as "others" as shown in Table 1.

\section{Prevalence of UTI according to patients" gender}

Females represented $71.4 \%$ of all positive urine cultures with an odds ratio of 1.863 (Table 2).

\section{The antibiogram of uropathogens recovered from patient samples}

Table 1 demonstrates that E. coli and Klebsiella spp. constitutes about $85 \%$ of the total isolates, which means that they are the main etiologic agents of $\mathrm{UTI}$, therefore, the study will focus on these isolates as the main findings. Aprominent finding is the high
Table 1. The distribution frequency of uropathogens isolates at the four governmental hospitals.

\begin{tabular}{|l|c|c|c|c|}
\hline \multirow{2}{*}{ Organism isolated } & \multicolumn{2}{|c|}{$\mathrm{n}=2,910$} & \multicolumn{2}{c|}{ Total } \\
\cline { 2 - 4 } & Frequency & $\%$ & $\mathrm{~N}$ & $\%$ \\
\hline Gram negative & & & & \\
\hline Escherichia coli & 1,743 & 59.9 & & \\
Klebsiella spp. & 725 & 24.9 & & \\
\hline Proteus spp. & 69 & 2.4 & 2672 & 91.8 \\
\hline Enterobacter spp. & 12 & 0.4 & & \\
\hline Pseudomonas spp. & 123 & 4.2 & & \\
\hline Gram positive & & & & \\
\hline Staphylococcus aureus & 41 & 1.4 & & \\
\hline Enterococcus spp. & 13 & 0.4 & 152 & 5.25 \\
\hline Streptococcus spp. & 98 & 3.4 & & \\
\hline Others & & & & \\
\hline Candida spp. & 70 & 2.4 & \multirow{2}{*}{86} & 2.95 \\
\hline Others & 16 & 0.5 & & \\
\hline Total & 2,910 & 100 & 2,910 & 100 \\
\hline
\end{tabular}

Table 2. Frequency of gender distribution of UTI cases from the four governmental hospitals in the Gaza Strip Palestine.

\begin{tabular}{|l|c|c|}
\hline \multirow{2}{*}{ Variable } & \multicolumn{2}{|c|}{ Gender distribution } \\
\cline { 2 - 3 } & Frequency & $\%$ \\
\hline Female & 2078 & 71 \\
Male & 832 & 29 \\
\hline Total & 2910 & 100 \\
\hline
\end{tabular}

resistance exhibited by uropathogens to penicillin group. The resistance to penicillin derivatives like ampicillin was $91 \%$ and amoxicillin was $90 \%$, for E. coli, and the resistance is $95 \%$ for Klebsiella spp. to the same antimicrobials Table 3. As shown in Table $3 \mathrm{E}$. coli is highly resistant to the cephalosporin group. Its resistance ranges from $41 \%$ to $60 \%$ against all of the cephalosporins. E. coli has the highest resistance against cephalexin (60\%) and the lowest resistance against ceftazidime (41\%). Klebsiella spp. has resistance (73\%) and (49\%) against the same antimicrobials, respectively. Also, Table 3 shows that the antibiotic resistance patterns of $E$. coli to the listed tested antibiotics. 
Table 3. Resistance pattern of uropathogens to antimicrobials at the four governmental hospitals in the Gaza Strip/Palestine during

\begin{tabular}{|c|c|c|c|c|c|c|c|c|c|c|c|c|c|c|c|c|c|}
\hline \multirow{2}{*}{ Antimicrobial } & \multirow{2}{*}{$\begin{array}{c}\text { Disk charge } \\
\text { Microgram }\end{array}$} & \multicolumn{2}{|c|}{ E. coli } & \multicolumn{2}{|c|}{ Klebsiella spp. } & \multicolumn{2}{|c|}{ Proteus spp. } & \multicolumn{2}{|c|}{ Enterobacter spp. } & \multicolumn{2}{|c|}{ Pseudomonas spp. } & \multicolumn{2}{|c|}{ S. aureus } & \multicolumn{2}{|c|}{ Enterococci } & \multicolumn{2}{|c|}{ Streptococcus spp. } \\
\hline & & $\mathrm{N}$ & $\%$ & N & $\%$ & N & $\%$ & N & $\%$ & N & $\%$ & $\mathrm{~N}$ & $\%$ & N & $\%$ & N & $\%$ \\
\hline Penicillin G & 6 & - & - & - & - & - & - & - & - & - & - & 3 & 75 & 4 & 36 & 1 & 100 \\
\hline Ampicillin & 10 & 553 & 91 & 245 & 95 & 27 & 90 & 5 & 100 & 30 & 100 & 3 & 60 & 3 & 30 & 5 & 100 \\
\hline Amoxicillin & 20 & 154 & 90 & 70 & 95 & 13 & 87 & 4 & 100 & 7 & 88 & 5 & 71 & 1 & 100 & 2 & 67 \\
\hline Cloxacillin & 5 & - & - & - & - & - & - & - & - & - & - & 5 & 50 & - & - & 19 & 79 \\
\hline Piperacillin & 100 & 148 & 74 & 90 & 79 & 2 & 50 & 1 & 50 & 19 & 79 & - & - & - & - & - & - \\
\hline Cephalexin & 30 & 540 & 60 & 279 & 73 & 21 & 68 & 2 & 67 & 42 & 91 & 2 & 15 & 4 & 67 & 29 & 74 \\
\hline Cefuroxime & 30 & 682 & 49 & 350 & 62 & 20 & 37 & 3 & 33 & 63 & 81 & 6 & 26 & 7 & 64 & 24 & 47 \\
\hline Cefotaxime & 30 & 474 & 44 & 267 & 60 & 10 & 24 & - & - & 29 & 43 & 2 & 67 & 4 & 57 & - & - \\
\hline Ceftazidime & 30 & 494 & 41 & 243 & 49 & 18 & 45 & 1 & 20 & 36 & 38 & 1 & 50 & 7 & 100 & - & - \\
\hline Ceftriaxone & 30 & 681 & 46 & 364 & 59 & 16 & 29 & 2 & 22 & 49 & 49 & 3 & 50 & 5 & 56 & - & - \\
\hline Cefazolin & 30 & 344 & 53 & 154 & 61 & 10 & 34 & 4 & 50 & 32 & 84 & 3 & 75 & 1 & 50 & - & - \\
\hline Doxycycline & 30 & 304 & 59 & 151 & 66 & 16 & 80 & 0 & 0 & 41 & 85 & 4 & 29 & - & - & 12 & 48 \\
\hline Amikacin & 30 & 15 & 2 & 14 & 4 & 1 & 3 & - & - & 5 & 8 & 1 & 17 & 5 & 63 & - & - \\
\hline Gentamicin & 30 & 321 & 23 & 172 & 29 & 20 & 38 & 4 & 40 & 23 & 24 & 8 & 30 & 7 & 54 & 25 & 48 \\
\hline Co-Trimoxazole & $1.25 / 23.75$ & 959 & 66 & 420 & 69 & 47 & 80 & 8 & 80 & 78 & 91 & 14 & 54 & 4 & 67 & 46 & 78 \\
\hline Nalidixic acid & 30 & 852 & 53 & 330 & 50 & 37 & 63 & 3 & 30 & 87 & 84 & 1 & 33 & 5 & 100 & 4 & 100 \\
\hline Ciprofloxacin & 5 & 450 & 35 & 198 & 38 & 16 & 34 & 4 & 40 & 36 & 36 & 12 & 38 & 7 & 70 & 45 & 63 \\
\hline Rifampicin & 5 & - & - & 1 & 50 & - & - & - & - & - & - & 1 & 3 & - & - & 27 & 36 \\
\hline Vancomycin & 30 & - & - & - & - & - & - & - & - & - & - & 8 & 21 & 2 & 18 & 17 & 18 \\
\hline Co-amoxiclav & $20 / 10$ & 45 & 73 & 17 & 68 & 5 & 63 & - & - & 3 & 75 & 13 & 45 & 1 & 50 & 32 & 42 \\
\hline Meropenem & 10 & 24 & 6 & 16 & 8 & 3 & 14 & 1 & 25 & 11 & 20 & 0 & 0 & 1 & 50 & - & - \\
\hline Nitrofurantoin & 300 & 20 & 15 & 24 & 44 & 2 & 50 & 0 & 0 & 8 & 73 & - & - & - & - & - & - \\
\hline $\begin{array}{l}\text { Piperacillin + } \\
\text { tazobactam }\end{array}$ & $100 / 10$ & 13 & 9 & 9 & 11 & 0 & 0 & 0 & 0 & 0 & 0 & - & - & - & - & - & - \\
\hline Total R \% & & 7082 & 45 & 3422 & 53 & 284 & 46 & 42 & 40 & 600 & 56 & 95 & 33 & 68 & 56 & 288 & 49 \\
\hline
\end{tabular}


Table 4. Antimicrobial resistance of uropathogens according gender at four governmental hospitals in the Gaza Strip- Palestine during 2019.

\begin{tabular}{|c|c|c|c|c|c|c|c|c|c|c|c|c|c|c|c|c|c|}
\hline \multirow{3}{*}{$\begin{array}{c}\text { Isolate } \\
\text { Antimicrobials }\end{array}$} & \multicolumn{4}{|c|}{ E. coli } & \multicolumn{4}{|c|}{ Klebsiella spp. } & \multicolumn{4}{|c|}{ Pseudomonas spp. } & \multicolumn{4}{|c|}{ Proteus spp. } & \multirow{3}{*}{ P. value } \\
\hline & \multicolumn{2}{|c|}{ Female } & \multicolumn{2}{|c|}{ Male } & \multicolumn{2}{|c|}{ Female } & \multicolumn{2}{|c|}{ Male } & \multicolumn{2}{|c|}{ Female } & \multicolumn{2}{|c|}{ Male } & \multicolumn{2}{|c|}{ Female } & \multicolumn{2}{|c|}{ Male } & \\
\hline & $\mathrm{N}$ & $\%$ & $N$ & $\%$ & $N$ & $\%$ & $\mathrm{~N}$ & $\%$ & $\mathrm{~N}$ & $\%$ & $\mathrm{~N}$ & $\%$ & $N$ & $\%$ & $\mathrm{~N}$ & $\%$ & \\
\hline Ampicillin & 428 & 90 & 125 & 94 & 150 & 94 & 95 & 97 & 12 & 100 & 18 & 100 & 19 & 86 & 8 & 100 & 0.164 \\
\hline Amoxicillin & 121 & 90 & 33 & 89 & 43 & 93 & 27 & 96 & 2 & 67 & 5 & 100 & 10 & 83 & 3 & 100 & 0.087 \\
\hline Piperacillin & 107 & 70 & 41 & 85 & 57 & 73 & 33 & 92 & 10 & 77 & 9 & 82 & 1 & 33 & 1 & 100 & 0.052 \\
\hline Cephalexin & 388 & 57 & 152 & 69 & 177 & 71 & 102 & 78 & 19 & 90 & 23 & 92 & 10 & 53 & 11 & 92 & 0.188 \\
\hline Cefuroxime & 483 & 45 & 199 & 62 & 219 & 59 & 131 & 66 & 28 & 76 & 35 & 85 & 10 & 29 & 10 & 53 & 0.282 \\
\hline Cefotaxime & 333 & 41 & 141 & 53 & 165 & 58 & 102 & 63 & 14 & 41 & 15 & 45 & 6 & 22 & 4 & 27 & 0.562 \\
\hline Ceftazidime & 330 & 37 & 164 & 52 & 158 & 48 & 85 & 53 & 17 & 35 & 19 & 40 & 12 & 43 & 6 & 50 & 0.105 \\
\hline Ceftriaxone & 473 & 42 & 208 & 56 & 233 & 56 & 131 & 65 & 18 & 38 & 31 & 60 & 10 & 28 & 6 & 30 & 0.272 \\
\hline Cefazolin & 248 & 50 & 96 & 64 & 97 & 58 & 57 & 68 & 14 & 78 & 18 & 90 & 6 & 27 & 4 & 57 & 0.242 \\
\hline Amikacin & 7 & 1 & 8 & 4 & 6 & 3 & 8 & 6 & 2 & 8 & 3 & 8 & 1 & 4 & 0 & 0 & 0.832 \\
\hline Gentamicin & 225 & 20 & 96 & 30 & 113 & 29 & 59 & 30 & 11 & 23 & 12 & 25 & 13 & 38 & 7 & 37 & 0.545 \\
\hline Doxycycline & 206 & 57 & 98 & 63 & 113 & 65 & 38 & 69 & 19 & 83 & 22 & 88 & 12 & 75 & 4 & 100 & 0.367 \\
\hline Co-Trimoxazole & 731 & 66 & 228 & 66 & 291 & 69 & 129 & 71 & 36 & 86 & 42 & 95 & 29 & 73 & 18 & 95 & 0.389 \\
\hline Nalidixic acid & 608 & 50 & 244 & 62 & 226 & 51 & 104 & 48 & 43 & 84 & 44 & 83 & 24 & 60 & 13 & 68 & 0.722 \\
\hline Ciprofloxacin & 292 & 30 & 158 & 48 & 136 & 38 & 62 & 38 & 21 & 40 & 15 & 31 & 9 & 30 & 7 & 41 & 0.299 \\
\hline Co-amoxiclav & 35 & 71 & 10 & 77 & 15 & 65 & 2 & 100 & 2 & 67 & 1 & 100 & 3 & 50 & 2 & 100 & 0.006 \\
\hline Meropenem & 15 & 6 & 9 & 8 & 12 & 9 & 4 & 7 & 5 & 17 & 6 & 23 & 3 & 20 & 0 & 0 & 0.572 \\
\hline Nitrofurantoin & 15 & 15 & 5 & 14 & 13 & 34 & 11 & 69 & 1 & 33 & 7 & 88 & 2 & 50 & 0 & 0 & 0.678 \\
\hline $\begin{array}{l}\text { Piperacillin + } \\
\text { tazobactam }\end{array}$ & 8 & 7 & 5 & 14 & 6 & 10 & 3 & 13 & 0 & 0 & 0 & 0 & 0 & 0 & 0 & 0 & 0.610 \\
\hline Total & 5053 & 43 & 2020 & 53 & 2230 & 51 & 1183 & 57 & 274 & 54 & 325 & 60 & 180 & 44 & 104 & 59 & 0.024 \\
\hline
\end{tabular}


Vol. 11 No. 1:4 doi: $10.3823 / 855$

\section{Antimicrobial resistance pattern of uropathogen according to patient's gender}

As shown in Table 4, among males, the resistance of $E$. Coli to antimicrobials was $10-15 \%$ higher than among females to the following antibiotics (piperacillin, cephalexin, cefotaxime cefuroxime, cefotaxime, ceftazidime, ceftriaxone cefazoline ciprofloxacin gentamicin, and nalidixic acid). Thetotal resistance of $E$. coli to all antimicrobials in females was $43 \%$, while in males it was $53 \%$. According to Klebsiella spp., among males, its resistance to the following antimicrobials (cephalexin, cefotaxime cefuroxime, cefotaxime, ceftazidime, ceftriaxone cefazoline, amikacin, doxycycline, ciprofloxacin gentamicin, cotrimoxazole, and nalidixic acid) was 3-9\% higher than in females. The total antimicrobial resistance of Klebsiella among female isolates is $51 \%$ while in males $57 \%$. Despite higher prevalence of UTI in the female than in males Table 2 , the resistance percentage against antimicrobial is higher in male than in the female. By conducting independent T-test for all antimicrobials, P. values is statistically significant only for co-amoxiclav $(P$. value $=0.006)$ and marginally significant for piperacillin (P. value $=0.052$ ) and the total antimicrobial resistance was statistically significant (P. value $=0.024)$ as shown in Table 4

\section{Antimicrobial resistance pattern of uropathogens according to patient's age}

As shown in Table 5, there was fluctuation in the resistance of the microorganisms against antimi-

Table 5. Percentage of antimicrobial resistance of all microorganisms at the four governmental hospitals distributed by age groups in the Gaza Strip-Palestine 2019.

\begin{tabular}{|c|c|c|c|c|c|c|c|c|c|c|}
\hline \multirow{3}{*}{ Antimicrobials } & \multicolumn{8}{|c|}{ Age groups } & \multirow{3}{*}{$\begin{array}{l}\text { ANOVA } \\
\text { P. value }\end{array}$} & \multirow{3}{*}{$\begin{array}{c}\text { Scheffe test } \\
\text { P. value }\end{array}$} \\
\hline & \multicolumn{2}{|c|}{$0-28$ day } & \multicolumn{2}{|c|}{ 29d-1 year } & \multicolumn{2}{|c|}{$1-12 y$} & \multicolumn{2}{|c|}{ Adult } & & \\
\hline & $\mathrm{R}$ & $\%$ & $\mathrm{R}$ & $\%$ & $\mathrm{R}$ & $\%$ & $R$ & $\%$ & & \\
\hline Cefuroxime & 22 & 47 & 99 & 46 & 463 & 44 & 577 & 66 & 0.300 & .012 \\
\hline Ampicillin & 30 & 97 & 86 & 91 & 485 & 90 & 277 & 96 & 0.046 & .010 \\
\hline Amoxicillin & 4 & 80 & 45 & 92 & 115 & 91 & 97 & 90 & 0.162 & .003 \\
\hline Piperacillin & 0 & 0 & 22 & 73 & 91 & 61 & 149 & 89 & 0.000 & .009 \\
\hline Cephalexin & 16 & 53 & 52 & 60 & 331 & 54 & 524 & 75 & 0.052 & .013 \\
\hline Cefotaxime & 13 & 43 & 57 & 39 & 279 & 37 & 441 & 62 & 0.035 & .077 \\
\hline Ceftazidime & 12 & 40 & 39 & 35 & 201 & 32 & 554 & 50 & 0.069 & .303 \\
\hline Ceftriaxone & 13 & 46 & 92 & 44 & 353 & 35 & 667 & 63 & 0.056 & .052 \\
\hline Cefazolin & 8 & 42 & 57 & 53 & 192 & 44 & 295 & 69 & 0.008 & .015 \\
\hline Amikacin & 1 & 3 & 6 & 4 & 14 & 2 & 22 & 6 & 0.036 & .416 \\
\hline Gentamicin & 7 & 16 & 24 & 12 & 146 & 14 & 407 & 41 & 0.000 & .002 \\
\hline Doxycycline & 6 & 67 & 7 & 64 & 12 & 52 & 504 & 62 & 0.097 & .274 \\
\hline Co-Trimoxazole & 26 & 60 & 131 & 67 & 564 & 63 & 859 & 73 & 0.436 & .002 \\
\hline Nalidixic acid & 17 & 39 & 78 & 36 & 394 & 38 & 835 & 71 & 0.000 & .004 \\
\hline Ciprofloxacin & 5 & 16 & 20 & 15 & 120 & 15 & 627 & 55 & 0.000 & .000 \\
\hline Co-amoxiclav & 2 & 100 & 11 & 69 & 37 & 71 & 68 & 46 & 0.815 & .134 \\
\hline Colistin & 0 & 0 & 17 & 85 & 106 & 60 & 9 & 28 & 0.000 & .870 \\
\hline Meropenem & 1 & 50 & 1 & 5 & 0 & 0 & 56 & 10 & 0.000 & .371 \\
\hline Nitrofurantoin & 1 & 25 & 2 & 33 & 4 & 13 & 49 & 29 & 0.984 & .567 \\
\hline $\begin{array}{l}\text { Piperacillin + } \\
\text { tazobactam }\end{array}$ & 0 & 0 & 5 & 21 & 11 & 10 & 6 & 5 & .011 & .896 \\
\hline
\end{tabular}


Vol. 11 No. 1:4 doi: $10.3823 / 855$ crobials in different age groups. ANOVA test was used to determine the significance of this variation. The result of ANOVA revealed that the fluctuation among age categories is statistically significant for number of antimicrobials (ampicillin, piperacillin, cefotaxime, cefazolin, amikacin, gentamicin, nalidixic acid, ciprofloxacin, and piperacillin + tazobactam) at P. value of $<0.05$ ). Two antimicrobials (cephalexin and ceftriaxone) at $P$. value of $<0.05$, while other antimicrobials were not statistically significant (e.g., cefuroxime, amoxicillin, ceftazidime, doxycycline, co-trimoxazole, co-amoxiclav, and nitrofurantoin). When Scheffe test was performed to test resistance variations among these age groups, it was found that there is a prominent correlation between age group 0-28 days and adult group with ( $P$. value of less than 0.05(for cefuroxime, ampicillin, amoxicillin, piperacillin, cephalexin, cefazolin, gentamicin, co-trimoxazole, nalidixic acid, and ciprofloxacin) Table $\mathbf{5}$.

\section{Antimicrobial resistance pattern of uropathogen according to both gender and age}

$E$. coli isolates were grouped according to age and gender, the difference is high between males and females in the age category of (0-28 days). Within the age category (29 days to 1 year), the resistance in females is higher than in males for most of the antimicrobials. However, in the age categories (1 year to 12 years) and adults, the resistance is higher in males to most of the antimicrobials than in females. As shown in Figure 1, E. coli resistance to antimicrobials among male isolates was higher than females isolate in most of the age categories. In the age category (0-28 days), $E$. coli isolates were resistant to amoxicillin is closed $100 \%$ in both genders. There is a difference in resistance of the E. coli against these antimicrobials (cephalexin, ceftazidime, ceftriaxone, and cefuroxime) in this age category about $30-40 \%$ between genders, however, both co-trimoxazole, and nalidixic acid show resistance in females, is higher than males in this age category. Among the age category (29 days to 1 year), E. coli in males is more resistant than in female against amoxicillin, cephalexin, and cefotaxime, while the resistance in female is higher than males against ceftazidime, gentamicin cephazolin, co-trimoxazole, nalidixic acid, and piperacillin Figure 2. In the age category (1 year to 12 years), $E$. coli resistance in males is higher than in females against piperacillin, cephalexin, cefuroxime, cefotaxime, ceftazidime, cefazoline, nalidixic acid, and ciprofloxacin. The resistance of $E$. coli in female isolates higher than male against amoxicillin and cotrimoxazole. The resistance of $E$. coli to gentamicin is almost the same Figure $\mathbf{3}$.

Figure 1: E. coli resistance percentage to antibiotics in males and females (0-28 days).

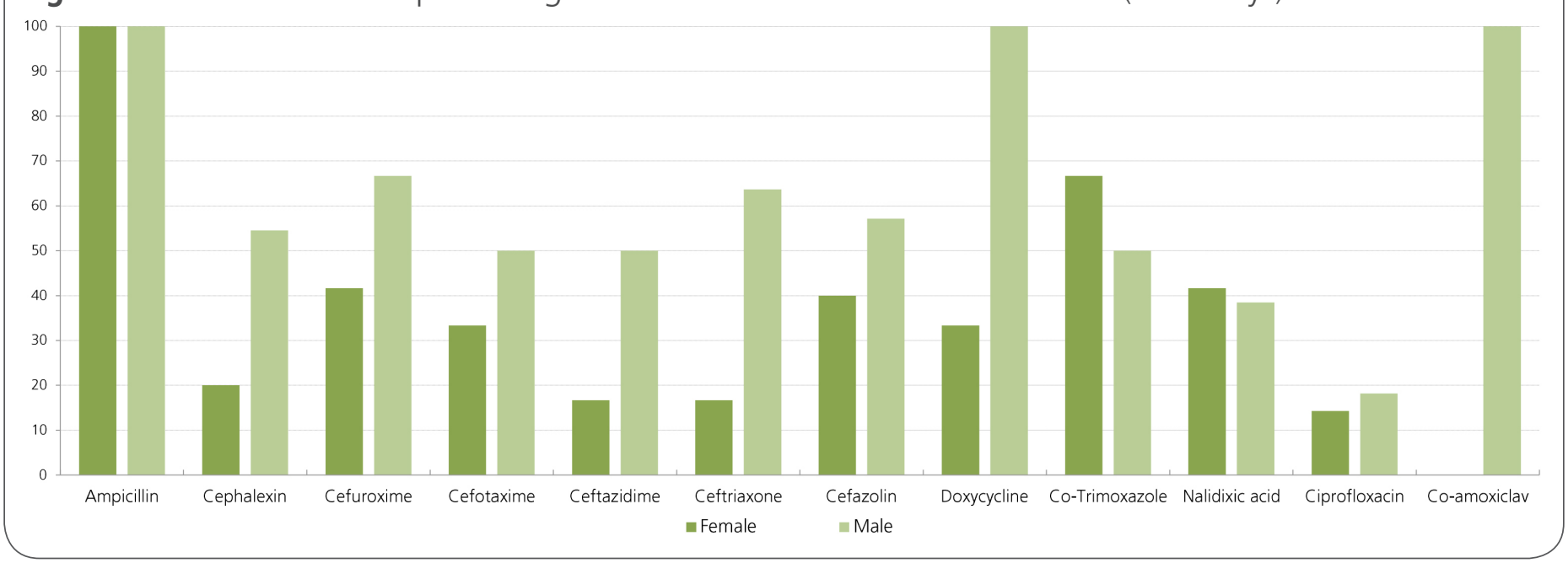




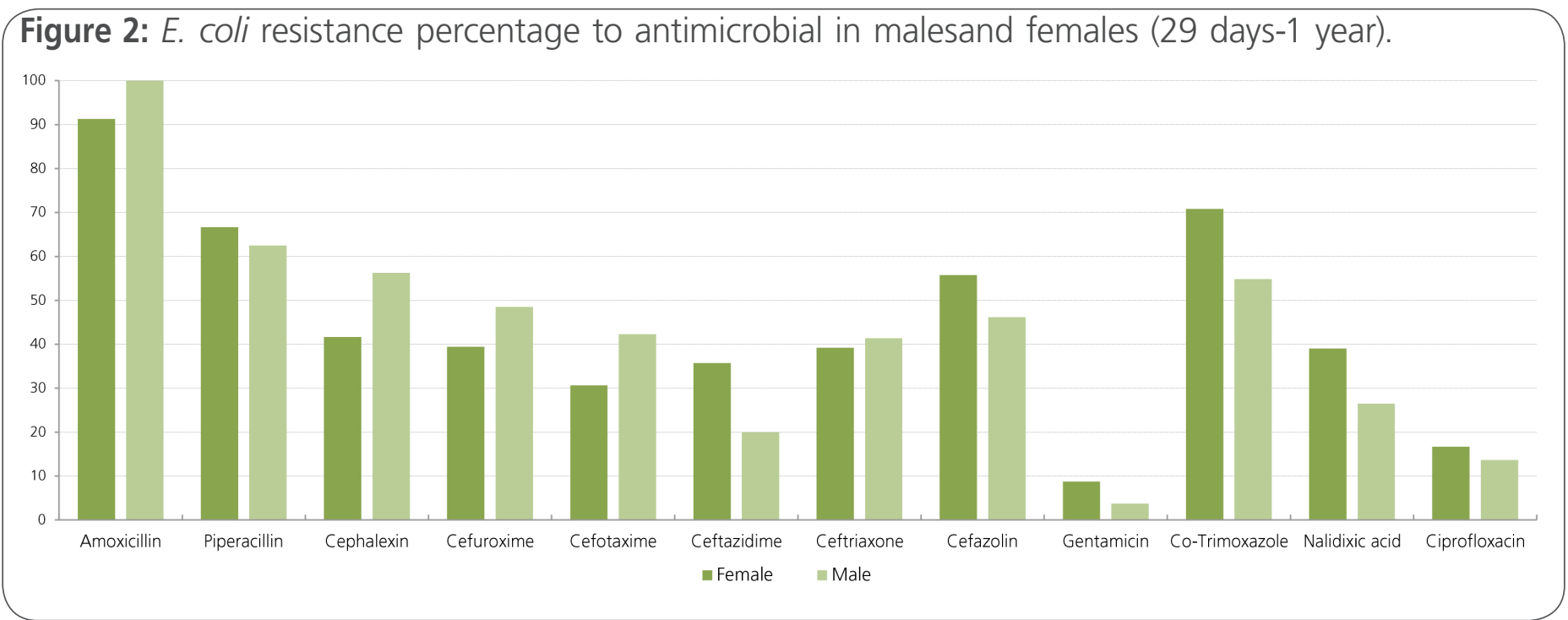

Figure 3: E. coli resistance percentage to antimicrobial in male female (1-12 years).

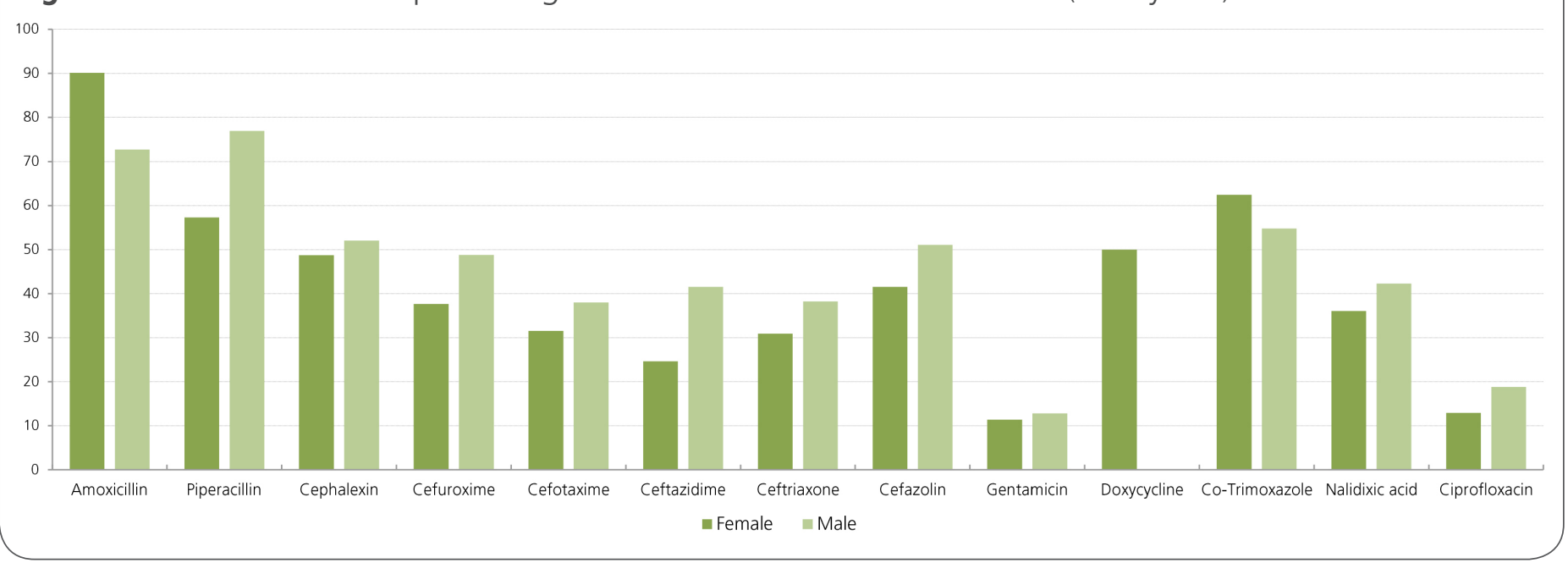

In the age category, (adults) the resistance of $E$. coli in male is higher than in female against the all of the antimicrobials (amoxicillin, piperacillin, cephalexin, cefuroxime, cefotaxime, ceftazidime, ceftriaxone, cefazolin, gentamicin, co-trimoxazole, nalidixic acid, ciprofloxacin) Figure 4.

\section{Multiple drug resistance in uropathogens}

Regarding to multidrug resistance definition [11], which is not yet agreed upon by the medical community. Many definitions are currently being used to characterize patterns. The most practical definition used for Gram-positive and Gram-negative bacteria is 'resistant to a single antibiotic in three or more antimicrobial classes [12], these antibiotic groups have been chosen according to lists suggested by professionals for isolates have no intrinsic factors for resistance to these antibiotics, and according to the standard operating procedures SOPs that is followed by the laboratory technicians at the governmental hospital in the Gaza Strip/Palestine, these antibiotics are ampicillin, cefuroxime, co-trimoxazole, ciprofloxacin, gentamicin, the isolates of $E$. coli and Klebsiella spp. as shown in Table 6, the observation is the resistance for a single antibiotic (ampicillin) from the penicillin group of $E$. coli is (91\%) and for Klebsiella spp. is (91\%) and by adding another antibiotic (cefuroxime) from cephalosporin group the 


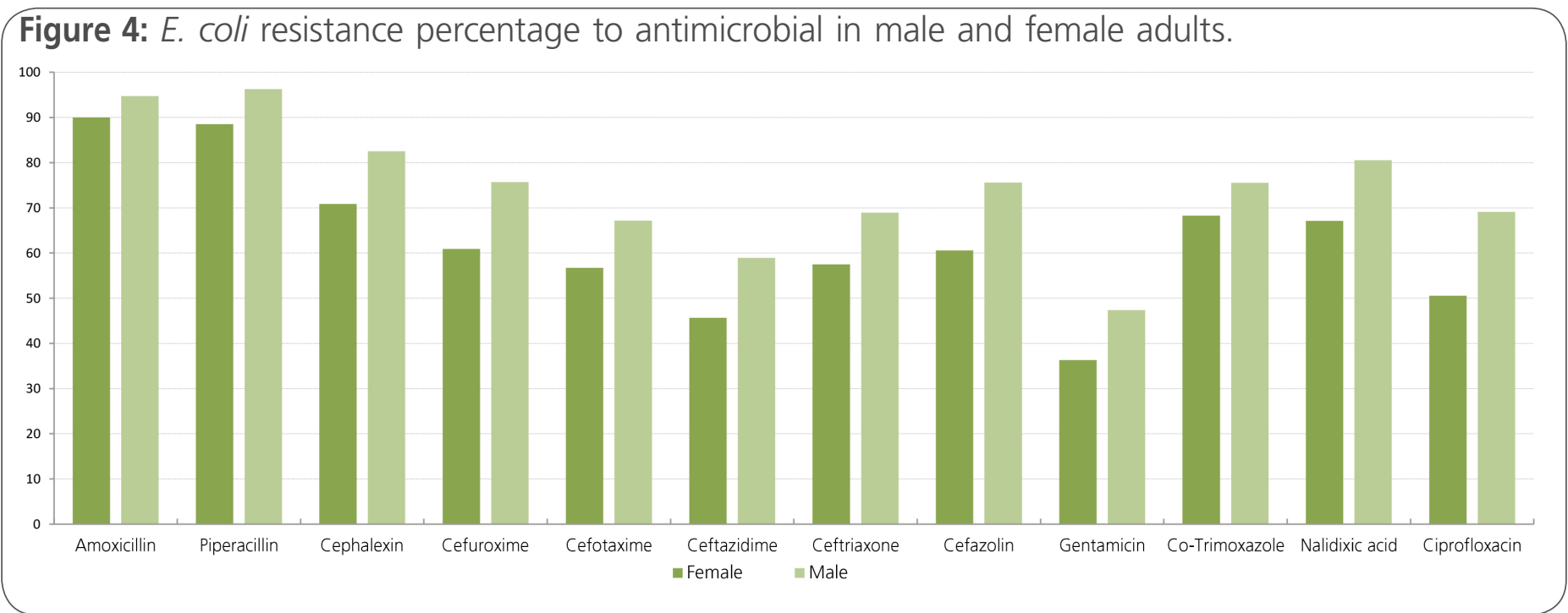

Table 6. Multidrug resistance percentage for uropathogens the same isolates for different antibiotic groups at governmental hospitals in Gaza Strip Palestine during 2019.

\begin{tabular}{|c|c|c|c|c|c|}
\hline \multirow[t]{2}{*}{ No } & \multirow[t]{2}{*}{ Antibiotic } & \multicolumn{2}{|c|}{$\begin{array}{l}\text { Escherichia } \\
\text { coli }\end{array}$} & \multicolumn{2}{|c|}{$\begin{array}{l}\text { Klebsiella } \\
\text { spp. }\end{array}$} \\
\hline & & N & $\%$ & N & $\%$ \\
\hline 1 & Ampicillin & 553 & 91 & 245 & 95 \\
\hline 2 & Ampicillin + Cefuroxime & 242 & 45 & 131 & 58 \\
\hline 3 & $\begin{array}{l}\text { Ampicillin + Cefuroxime } \\
+ \text { Co-Trimoxazole }\end{array}$ & 162 & 37 & 90 & 53 \\
\hline 4 & $\begin{array}{l}\text { Ampicillin + Cefuroxime } \\
+ \text { Co-Trimoxazole+ } \\
\text { Ciprofloxacin }\end{array}$ & 55 & 18 & 33 & 31 \\
\hline 5 & $\begin{array}{l}\text { Ampicillin + Cefuroxime } \\
+ \text { Co-Trimoxazole+ } \\
\text { Ciprofloxacin + } \\
\text { Gentamicin }\end{array}$ & 29 & 11 & 21 & 22 \\
\hline
\end{tabular}

resistance percentage for isolates for penicillin and cefuroxime for the same isolates is $(45 \%)$ for $E$. coli and (58\%) for Klebsiella spp., after adding the third antibiotic (Co-Trimethoprim) from aminoglycoside group the resistance for the same isolates for these antibiotic groups is (37\%) for E. coli and (53\%) for Klebsiella spp., after addition of the fourth antibiotic group fluoroquinolone (ciprofloxacin) and according to the definition, the multidrug resistance is (18\%) for E. coli and (31\%) for Klebsiella spp., after addition of the fifth antibiotic group (Gentamicin) multidrug resistance is (11\%) for E. coli and (22\%) for Klebsiella spp., from these findings multidrug resistance was detected in uropathogens, (37\%) for E. coli and (53\%) for Klebsiella spp. the same isolates by three different antibiotics in different groups. This is alarming and should be considered by any antibiotic stewardship programs. (Table 6)

\section{Discussion}

This study aimed to determine the resistance pattern of uropathogens to antimicrobials and to explore the MDR at the governmental hospitals in the Gaza Strip/Palestine. Uropathogens were isolated from $24.5 \%$ of the requested urine cultures. $E$. coli constituted the majority of isolates with $59.9 \%$, followed by Klebsiella spp. (24.9\%) and all Gramnegative constituted $91 \%$ of the isolates. Females represented by $71.4 \%$ of all positive urine cultures. E. coli resistance rate to penicillin derivatives like ampicillin was $91 \%$ and amoxicillin $90 \%$, while resistance rate of Klebsiella spp. was 95\% for the same antimicrobials. $E$. coli is highly resistant to the cephalosporin group. Its resistance is between $41 \%$ and $60 \%$ against all of the cephalosporines, Klebsiella spp. has resistance (73\%) and (49\%) against the same antimicrobials, respectively. Both of $E$. coli 
and Klebsiella have the minimal resistance against amikacin, which are (2\%) and (4\%), respectively. The resistance percentage against the tested antimicrobials is higher in male than in the female. Regarding to our study, MDR in Gaza governmental hospitals is (37\%) for E. coli and (53\%) for Klebsiella spp., respectively.

We demonstrated very high prevalence of Gramnegative isolates and this in agreement with a study in Lahore, Pakistan conducted between December 2018 and June 2019, which reported that prevalence of $92 \%$ Gram negative [13]. Another study in Ethiopia reveled that Gram-negative bacteria caused $90 \%$ of UTI cases while gram-positive bacteria cause only $10 \%$ of the cases [14]. In North Ethiopia, E. coli prevalence in UTI was $60.29 \%$ and Klebsiella spp. prevalence was $5.88 \%$ in a study conducted from 2002 to 2011 [15]. Another study reported $79 \%$ E. coli prevalence in UTI in Luxembourg [16] which higher than our findings.

In Sarajevo, E. coli had the highest antimicrobial resistance to trimethoprim/sulfamethoxazole (38.61\%), followed by amoxicillin/clavulanic acid (19.62\%), ciprofloxacin (9.49\%), genta-micin $(8.86 \%)$, cephalexin (8.23\%), nitrofurantoin $(8.23 \%)$, cefuroxime $(7.52 \%)$, ceftazidime $(6.33 \%)$, cefuroxime (89.87\%), amikacin (4.43\%) [17]. These findings are lower than our findings. A study from Iraq in 2016 demonstrated resistance pattern for $E$. coli similar to this study. The resistance was $100 \%$ for penicillin and 52\% for cephalosporin [18]. In Saudi Arabia, the resistance of Klebsiella spp. was 91\% against ampicillin, 57\% against cefotaxime, and $46 \%$ against ceftazidime [19]. Another study from India revealed that $E$. coli resistance against amikacin was (16-38\%), followed by cefotaxime (38-40\%), gentamicin (42-68\%), ciprofloxacin (54$73 \%)$, and co-trimoxazole (74-90\%) during the period between 2000 and 2004 [20].

A study in Saudi Arabia found that the resistance of $E$. coliagainst meropenem was $7 \%$, amikacin (9\%) and gentamicin (18\%) [19]. In south Iran, a study conducted in 2019 found that the resistance of $E$. coli against nalidixic acid was $71.9 \%$ [21]. In Rajasthan, a 2012 study recorded an overall resistance of $E$. coli against nalidixic acid, norfloxacin, and ciprofloxacin as (94\%), (77\%), and 74.75\%, respectively [22].

A retrospective study investigated 16,883 urine culture samples conducted in Palestine between 2001 and 2013, demonstrated that Gram-negative uropathogens showed good susceptibility towards ciprofloxacin, ofloxacin, norfloxacin, cefuroxime, nitrofurantoin, sulphathiazole/trimethoprim, nalidixic acid, and amoxicillin/clavulanic acid, (76.1\%, 71.8\%, $72.9 \%, 64 \%, 67.5 \%, 48.7 \%, 57.6 \%, 42.1 \%$, respectively) [23].

In Turkey, a retrospective study, evaluated causative agents and antimicrobial resistance in urine isolates from the positive community period between 2014 and 2016, it revealed that the resistance of $E$. coli during these years against co-trimoxazole was (29.8\%) [24]. In addition, higher resistance rates against ampicillin Bangladesh 2020 found (100\%) [25].

These variations among various studies from different countries may be due to different geographical settings, variations in use or misuse of antimicrobials, different treatment protocols, and study sample size among many other causes. Resistance varies over time and many of the above listed studies were conducted in different settings and times.

According to 2020 CDC update, a microorganism protects itself from antibiotic in various ways.K. pneumoniae produce enzymes called carbapenems, which break down carbapenem drugs and most other beta-lactam drugs.E. coli bacteria with the mcr-1 gene can add a compound to the outside of the cell wall so that the drug colistin cannot latch onto it [26].

Several risk factors are associated with UTIs, including gender. The shorter distance to the bladder in women makes it easier for bacterial colonizers to reach it [27]. Male patients, have a lower risk of contracting uncomplicated UTIs but are more prone 
to contracting complicated or MDR infections [28]. Our data reflect the severe nature of UTI in men, in which infections are caused by MDR microorganisms

A study conducted in Pakistan in 2008 gave similar results, it reported variations among age groups with regard to resistance and it appeared that in the case of males, E. coli isolates were susceptible to nitrofurantoin in age groups $0+, 50+$, and $70+$, while in age groups $20+, 30+$ and $60+$ hundred percent resistance was recorded. E. coli. In females, showed no resistance to nitrofurantoin in age groups $10+$, $50+$, and $70+$, while it showed $33 \%$ resistance in age groups $0+, 20+$, and $30+$. E. coli isolates from age groups $50+$ and $70+$ both in males and females were found to be $100 \%$ susceptible to the nitrofurantoin [29].

One study concluded that antibiotics that target DNA synthesis result in a progressively higher number of resistant isolates among the older population. The results emphasize the importance of patient age on antibiotic selection as a preventive measure to reduce the rate of resistant infections in each susceptible population. This pattern suggests that physicians should take into consideration patient age as another factor in determining the best antibiotic regiment with the aim of curtailing the emergence of newer resistant phenotypes in the future [30].

A study in southwest Washington in 2013 showed statistically significant differences between males and females in the age-specific susceptibilities of $E$. coli to ampicillin, co-amoxiclav, ciprofloxacin, and nitrofurantoin. Urinary $E$. coli isolates from male patients tend to exhibit increased antimicrobial resistance than isolates from female patients. Despite the statistical significance of time trends and differences in age-specific susceptibilities, the magnitude of these differences was generally less than 5\% and thus may not represent clinically meaningful differences. The exception was susceptibility to amoxicillin-clavulanate, where susceptibility was roughly $10 \%$ lower in males aging 18 to 64 years than females in the same age group [31]. A 10 year study of community UTI in Portuguese patients also identified differences in antimicrobial susceptibility according to patient gender. The authors reported that urinary isolates of $E$. coli were significantly more resistant to fluoroquinolones, penicillin's, nitrofurantoin, and first and secondgeneration cephalosporins among men compared to women [32]. Another study focused on pediatric patients, also identified significantly higher resistance to co-trimoxazole and ciprofloxacin in male versus female patients [33].

The NAUTICA (the North American Urinary Tract Infection Collaborative Alliance) surveillance study of outpatient UTIs, reported greater antimicrobial resistance to ciprofloxacin, levofloxacin, and co-trimoxazole among all urinary isolates from U.S. and Canadian male patients [34]. In the CANWARD (Canadian Ward) Surveillance Study, antimicrobial susceptibility among all E. coli isolates (not limited to urine isolates) collected from Canadian tertiary medical centers were compared and resistance was also observed to be significantly higher to ciprofloxacin, levofloxacin, and co-trimoxazole in isolates collected from male patients versus female patients [35].

Most studies of urinary tract infections (UTIS) focus on female patients because of the higher incidence in women than in men. For this reason, most UTI guidelines are based on studies performed on women, despite the obvious genito-urinary differences [36]. In men, UTIs become increasingly frequent with age and functional disability, but bacteriuria in elderly men tends to be intermittent, episodic, and more complex to diagnose. It is generally recognized that treatment recommendations for women are not usually appropriate for men. However, guidelines or recommendations for the management of men are mostly based on studies in women, children and institutionalized elderly people of both sexes [3]. 
A study conducted in Arabia Saudi in 2018 showed a slightly high level of multidrug resistance among the tested isolates if compared with our results with $(67 \%)$ of all $E$. coli isolates showing the MDR phenotype. Of these isolates, (26.86\%) isolates were resistant to 3 antibiotic groups $(25.37 \%)$ were resistant to 4 antibiotic groups, and (14.92\%) were resistant to 5 antibiotic groups [37]. A study conducted in Jordan, reviled that there is high percentage of uropathogenic $E$. coli isolates from outpatients and hospitalized Jordanian patients in Amman area, is multidrug resistant to at least 3 antibiotics [38].

\section{Conclusion}

The main etiologic agents for UTI sare $E$ coli and klebsiella spp. Penicillin is no longer be suitable treatment for UTI. Both age and gender are risk factors in both infection and resistance pattern. Antimicrobial stewardship program should be established under the $\mathrm{MoH}$ supervision, physicians should minimize the empirical use of antimicrobials in nonlife-threatening infection and rely more on culture and sensitivity for specific treatment. This type of study should be performed regularly to monitor the resistance of microorganisms to antimicrobials, not only for UTI but also for all types of infections for humans and animals as well to find the proper way to minimize multidrug resistance.

\section{References}

1. Tajbakhsh E, Tajbakhsh S, Khamesipour F. Isolation and molecular detection of Gram negative bacteria causing urinary tract infection in patients referred to Shahrekord hospitals, Iran. IRCMJ2015; 17(5).

2. American College of Obstetricians and Gynecologists. ACOG Practice Bulletin No. 91: Treatment of urinary tract infections in nonpregnant women. OB/GYN 2008; 111(3):785-94..

3. Hummers-Pradier E, Ohse AM, Koch M, Heizmann WR, Kochen MM. Urinary tract infection in men. Int J Clin Pharmacol Ther 2004; 42(7):360-6.

4. Momoh AR, Orhue PO, Idonije OB, Oaikhena AG, Nwoke EO, Momoh AA. The antibiogram types of Escherichia coli isolated from suspected urinary tract infection samples. J Microbiol Biotech Res 2011; 1(3):57-65.

5. Mohammed MA, Alnour TM, Shakurfo OM, Aburass MM. Prevalence and antimicrobial resistance pattern of bacterial strains isolated from patients with urinary tract infection in Messalata Central Hospital, Libya. Asian Pac JTrop 2016; 9(8):771-6.

6. Fasugba O, Gardner A, Mitchell BG, Mnatzaganian G. Ciprofloxacin resistance in community-and hospital-acquired Escherichia coli urinary tract infections: a systematic review and meta-analysis of observational studies. BMC Infect Dis2015; 15(1):1-6.

7. Pallett $A$, Hand K. Complicated urinary tract infections: practical solutions for the treatment of multiresistant Gram-negative bacteria. JAntimicrobChemother2010; 65(suppl_3):iii25-33.

8. World Health Organization. Antimicrobial resistance global report on surveillance: 2014 summary. WHO2014.

9. Kanapathipillai R, Malou N, Baldwin K, Marty P, Rodaix C, Mills C, Herard P, Saim M. Antibiotic resistance in Palestine: an emerging part of a larger crisis. Bmj2018; 363.

10. CLSI, Performance Standards for Antimicrobial Susceptibility Testing, Clinical and Laboratory Standards Institute (CLSI), Wayne, PA, USA 2018.

11. Shenoy KA, Jyothi EK, Ravikumar R. Phenotypic identification \& molecular detection of blandm-1 gene in multidrug resistant Gram-negative bacilli in a tertiary care centre. Indian J Med Res 2014; 139:625-31.

12. Magiorakos AP, Srinivasan A, Carey RT, Carmeli $Y$, Falagas MT, Giske CT, Harbarth S, Hindler JT, Kahlmeter G, Olsson-Liljequist B, Paterson DT. Multidrug-resistant, extensively drug-resistant and pandrug-resistant bacteria: an international expert proposal for interim standard definitions for acquired resistance. CMI 2012; 18(3):268-81.

13. Asmat U, Mumtaz MZ, Malik A. Rising prevalence of multidrugresistant uropathogenic bacteria from urinary tract infections in pregnant women. J Taibah Univ Medical Sci 2020.

14. Seifu WD, Gebissa AD. Prevalence and antibiotic susceptibility of Uropathogens from cases of urinary tract infections (UTI) in Shashemene referral hospital, Ethiopia. BMC Infect Dis2018; 18(1):1-9.

15. Abejew AA, Denboba AA, Mekonnen AG. Prevalence and antibiotic resistance pattern of urinary tract bacterial infections in Dessie area, North-East Ethiopia. BMC Res Notes. 2014; 7(1):1-7.

16. Saperston KN, Shapiro DJ, Hersh AL, Copp HL. A comparison of inpatient versus outpatient resistance patterns of pediatric urinary tract infection.J Urol2014; 191(5):1608-13.

17. Abduzaimovic A, Aljicevic M, Rebic V, Vranic SM, Abduzaimovic $K$, Sestic $S$. Antibiotic resistance in urinary isolates of Escherichia coli. Materia Socio-Medica 2016; 28(6):416. 
Vol. 11 No. 1:4 doi: $10.3823 / 855$
18. Polse R, Yousif S, Assafi M. Prevalence and antimicrobial susceptibility patterns of uropathogenic $E$. coli among people in Zakho, Iraq. Int J Res Med Sci 2016; 4(4):1219-23.

19. Taher I, Almaeen A, Aljourfi H, Bohassan E, Helmy A, El-Masry E, Saleh B, Aljaber N. Surveillance of antibiotic resistance among uropathogens in Aljouf region northern Saudi Arabia. IJM 2019; 11(6):468

20. Gupta N, KundraS, Sharma A, Gautam V, Arora DR. Antimicrobial susceptibility of uropathogens in India. J Infect Dis Antimicrob Agents. 2007; 24(1):13-8.

21. Malekzadegan $Y$, Rastegar E, Moradi M, Heidari H, EbrahimSaraie HS. Prevalence of quinolone-resistant uropathogenic Escherichia coli in a tertiary care hospital in south Iran. Infect Drug Resist 2019; 12:1683.

22. Sood S, Gupta R. Antibiotic resistance pattern of community acquired uropathogens at a tertiary care hospital in Jaipur, Rajasthan. IJCM: official publication of IAPSM 2012; 37(1):39.

23. Zaid AM. Distribution of bacterial uropathogens and their susceptibility patterns over twelve years (2001-2013) in Palestine. IAJAA 2014; 3(4).

24. Gunduz S, Altun HU. Antibiotic resistance patterns of urinary tract pathogens in Turkish children. Glob Health Res Policy 2018; 3(1):1-5

25. levy S, Islam M, Sobur M, Talukder M, Rahman M, Khan MF. Molecular detection of avian pathogenic Escherichia coli (APEC) for the first time in layer farms in Bangladesh and their antibiotic resistance patterns. Micro 2020; 8(7):1021.
26. CDC, How Antibiotic Resistance Happens Centers for Disease Control and Prevention, National Center for Emerging and Zoonotic Infectious Diseases 2020. Access date 30/1/2021

27. Foxman B. The epidemiology of urinary tract infection. Nat Rev Urol 2010; 7(12):653-60.

28. Schaeffer AJ, Nicolle LE. Urinary tract infections in older men. NEJM 2016; 374(6):562-71.

29. Bashir MF, Qazi Jl, Ahmad N, Riaz S. Diversity of urinary tract pathogens and drug resistant isolates of Escherichia coli in different age and gender groups of Pakistanis. Trop J Pharm Res 2008; 7(3):1025-31.

30. Garcia A, Delorme T, Nasr P. Patient age as a factor of antibiotic resistance in methicillin-resistant Staphylococcus aureus. J Med Microbiol 2017; 66(12):1782-9.

31. McGregor JC, Elman MR, Bearden DT, Smith DH. Sex-and agespecific trends in antibiotic resistance patterns of Escherichia coli urinary isolates from outpatients. BMC FamPract2013; 14(1):1-5.

32. Linhares I, Raposo T, Rodrigues A, Almeida A. Frequency and antimicrobial resistance patterns of bacteria implicated in community urinary tract infections: a ten-year surveillance study (2000-2009). BMC InfectDis 2013; 13(1):1-4.

33. Edlin RS, Shapiro DJ, Hersh AL, Copp HL. Antibiotic resistance patterns of outpatient pediatric urinary tract infections. J Urol 2013; 190(1):222-7.

34. Zhanel GG, Hisanaga TL, Laing NM, DeCorby MR, Nichol KA, Palatnick LP, Johnson J, Noreddin A, Harding GK, Nicolle LE, Hoban DJ. Antibiotic resistance in outpatient urinary isolates: final results from the North American Urinary Tract Infection Collaborative Alliance (NAUTICA). Int J Antimicrob Agents 2005; 26(5):380-8.

\section{Publish in The International} Arabic Journal of Antimicrobial Agents

The Journal is an open access peer-reviewed journal that publishes scientific papers about all aspects of antimicrobials. The journal will publish original research articles, reviews, brief reports and case reports dealing with basic and clinical antibacterial agents, antiviral, antiprotozoals, antituberculuous, antifungal and antihelminthes agents. All manuscripts must be prepared in English, and are subject to a rigorous and fair peer-review process. Accepted papers will immediately appear online. The journal aims to advance the knowledge, attitude and the research of chemotherapy in the Arabic world in cooperation with international, national scientific and public societies as well as research centers with similar aims and objectives. 